\title{
Frequency specific modulation of human somatosensory cortex
}

\author{
Matteo Feurra, ${ }^{1,2 *}$, Walter Paulus ${ }^{3}$, Vincent Walsh ${ }^{1}$ and Ryota Kanai ${ }^{1}$ \\ 1 Institute of Cognitive Neuroscience, Department of Psychology, University College London, London, UK \\ 2 Department of Neuroscience, Neurology Section, University of Siena, Siena, Italy \\ ${ }^{3}$ Department of Clinical Neurophysiology, Georg-August University, Göttingen, Germany
}

Edited by:

Gregor Thut, University of Glasgow, UK

Reviewed by:

Carlo Miniussi, University of Brescia,

Italy

Lisa Marshall, University of Luebeck,

Germany

${ }^{*}$ Correspondence:

Matteo Feurra, Department of

Neuroscience, Neurology Section,

University of Siena, Policlinico le Scotte,

Viale Bracci, l-53100 Siena, Italy.

e-mail: matfeu@googlemail.com
Oscillatory neuronal activities are commonly observed in response to sensory stimulation. However, their functional roles are still the subject of debate. One-way to probe the roles of oscillatory neural activities is to deliver alternating current to the cortex at biologically relevant frequencies and examine whether such stimulation influences perception and cognition. In this study, we tested whether transcranial alternating current stimulation (tACS) over the primary somatosensory cortex (SI) could elicit tactile sensations in humans in a frequency-dependent manner. We tested the effectiveness of tACS over SI at frequency bands ranging from 2 to $70 \mathrm{~Hz}$. Our results show that stimulation in alpha $(10-14 \mathrm{~Hz})$ and high gamma $(52-70 \mathrm{~Hz})$ frequency range produces a tactile sensation in the contralateral hand. A weaker effect was also observed for beta $(16-20 \mathrm{~Hz}$ ) stimulation. These findings highlight the frequency dependency of effective tACS over SI with the effective frequencies corresponding to those observed in previous electroencephalography/magnetoencephalography studies of tactile perception. Our present study suggests that tACS could be used as a powerful online stimulation technique to reveal the causal roles of oscillatory brain activities.

Keywords: tACS, somatosensory cortex, frequencies, tactile

\section{INTRODUCTION}

In humans, oscillations at different frequencies reflect different behaviors and cognitive processes (Engel et al., 2001) as typically measured by electroencephalography (EEG) and magnetoencephalography (MEG; Llinas and Steriade, 2006). These are due to synchronous neuronal activity within the neocortex and their interactions with thalamic and hippocampal circuitry (Fries, 2005). To establish causal functions of oscillatory neuronal activities, one promising approach is to examine whether stimulation of the cortex with physiologically relevant frequencies affects behavior and cognition (Thut and Miniussi, 2009). However, only a handful of studies have examined the effects of oscillatory current stimulation on the human brain (Marshall et al., 2006; Kanai et al., 2008; Pogosyan et al., 2009).

Transcranial alternating current stimulation (tACS) is still a largely unexplored technique and volume conduction effects are not wholly understood (Schwiedrzik, 2009; Kanai et al., 2010; Paulus, 2010; Schutter and Hortensius, 2010; Zaghi et al., 2010). A recent study showed that tACS of primary visual cortex (V1) induces visual phenomena such as a percept of continuously flickering light (phosphene), depending on the frequency of stimulation (Kanai et al., 2008). Critically, when visual phosphenes are induced by tACS, weak current still reaches the peri-orbital regions via volume conduction (Schutter and Hortensius, 2010), which could directly stimulate the retina rather than the visual cortex. Furthermore, the frequency specificity and its interaction with lighting conditions mirror known relationships between stimulation frequency and retinal sensitivity (Schwiedrzik, 2009). Specifically, ACS of the retina was most effective in the beta range (Rohracher, 1935) and the effective frequency for the retinal phosphene shifts toward lower frequencies after dark adaptation (Schwarz, 1947). This pattern of results is exactly what was found with tACS over the visual cortex. Thus, these earlier findings raise a serious concern regarding the origin of the phosphene evoked by tACS.

In this respect, a recent study by Zaehle et al. (2010) is important, as they provided direct physiological evidence of interaction between tACS and ongoing alpha oscillation in the occipital region. When tACS was delivered at the individual alpha-frequency adjusted for individuals for a prolonged period, entrainment of the EEG amplitude in this frequency was observed (Zaehle et al., 2010). Moreover, cortical excitability of the visual cortex as measured by the thresholds for transcranial magnetic stimulation (TMS)evoked phosphenes, exhibits frequency dependency whereby $20 \mathrm{~Hz}$ tACS over the visual cortex enhances the sensitivity of the visual cortex (Kanai et al., 2010). While these findings suggest that tACS indeed interacts with physiological properties of the visual cortex, the question of whether tACS has the capacity to induce subjective cortically induced sensations in a frequency-dependent manner remains unresolved.

In this study, we examined whether tACS could induce a frequency-dependent sensation in another sensory modality touch, because induction of tactile sensations far away from the stimulation electrodes, can provide independent support about the issue that induction of sensory perception is possible by cortical alternate current stimulation (Schwiedrzik, 2009; Schutter and Hortensius, 2010). Specifically, we chose to target the right somatosensory cortex (SI), because the peripheral nerve endings are distant from the stimulation site for the somatosensory 
cortex, and any spatially specific induction of a tactile sensation would indicate the effectiveness of cortical stimulation by tACS. Furthermore, to establish the relevance of particular stimulation frequencies, we tested whether tactile sensations could be observed only at a specific stimulation frequency.

\section{MATERIALS AND METHODS SUBJECTS}

Fourteen naïve healthy volunteers (mean age: 28.9; SD: 4.5) took part in the study. All subjects gave informed consent in accordance with the Declaration of Helsinki and the procedures had the approval of the ethical committee of University College London. Subjects had no metallic implants, previous history of any neurological disorders, medication, or substance abuse.

\section{SITE LOCALIZATION WITH TMS}

Transcranial magnetic stimulation was used to localize the somatosensory hand area in the postcentral gyrus that represents the contralateral hand (Yousry et al., 1997). TMS was delivered by a Magstim Super Rapid stimulator (Magstim Company, UK) via a 70-mm figure-of-eight shaped coil. During the site localization as well as the main tACS experiment, subjects were asked to lie supine on a medical chair and were told to relax.

For localizing the hand area in SI, we first determined the hot spot for the primary motor cortex (M1) at different intensities until we were able to induce a visible twitch in the contralateral (i.e., left) first dorsal interosseous. Stimulation intensity was then set at $120 \%$ of the participant's active motor threshold. From this hotspot, we then gradually moved the coil position in the posterior direction in a step size of $0.5 \mathrm{~cm}$, delivering four pulses per step. Participants were asked to report what kind of sensation they felt in the hand after the delivery of each TMS pulse. Participants reported a tactile sensation in their contralateral hand without visible TMS-triggered twitches typically near the C4 position of the international 10-20 system. Near the C4 position where motor responses were not observed, we determined the hot spot at which participants reported the strongest tactile sensation. This point was marked for tACS montage.

\section{TRANSCRANIAL ALTERNATING CURRENT STIMULATION}

Transcranial alternating current stimulation was delivered by a battery driven, constant current stimulator (NeuroConn $\mathrm{GmbH}$, Ilmenau, Germany) through a pair of surface saline-soaked sponge electrodes. We used a $3 \mathrm{~cm} \times 4 \mathrm{~cm}$ electrode over the right somatosensory cortex to increase focality (Nitsche et al., 2007) of somatosensory cortex stimulation whereas a standard $5 \mathrm{~cm} \times 7 \mathrm{~cm}$ electrode was used as reference. The reference electrode was placed over P3 (left posterior parietal cortex) which is far enough from the stimulation electrode (Nitsche et al., 2007). The experiment was conducted with impedance between the electrodes around $10 \mathrm{k} \Omega$ or below to minimize skin sensation. The waveform of the stimulation was sinusoidal and there was no DC offset. The current was delivered for $5 \mathrm{~s}$ per trial with an intensity of $1500 \mu \mathrm{A}$ ranging from 2 to $70 \mathrm{~Hz}$ frequencies. The order of stimulation frequencies was randomized across trials. The maximum current density at the stimulation electrode corresponded to $62.5 \mu \mathrm{A} / \mathrm{cm}^{2}$.

\section{TASK}

To familiarize our participants with the tactile sensation induced by $1500 \mu \mathrm{A}$ tACS, we delivered tACS at both low (4-12 Hz) and high $(50-80 \mathrm{~Hz})$ frequencies, which we found to be effective at inducing tactile sensation in our less systematic pilot study. Once they identified subtle tactile sensations in their hand, the stimulation was repeated and they were asked to describe the sensation verbally. They typically reported the feeling of tingles on their fingertips or the impression of some stings (without any pain sensations) on their hand without any physical stimulation actually being acted on the skin. Each trial of stimulation lasted $5 \mathrm{~s}$ and participants were instructed to give a rating for the strength of tactile sensation between 0 and 3 . We asked the participants to give a 0 rating when there was nothing noticeable during stimulation, a rating of 1 when they thought there might be a faint tactile sensation, 2 when they felt a tactile sensation, and 3 when the sensation was clear and strong. Subjects usually reported the tactile sensation lasting a couple of seconds.

During the experiment, we delivered tACS at a current intensity of $1500 \mu \mathrm{A}$ and tested each frequency in a step size of $2 \mathrm{~Hz}$ from 2 to $70 \mathrm{~Hz}$ ( 35 trials). The order of stimulation frequency was randomized across trials. Subjects were blind to the stimulation frequency that was used for each trial. Subjects were asked to give a tactile sensation rating according to the criterion for the training session. Once the first session was finished, $10 \mathrm{~min}$ later, we began the main experiment using the same protocol of the training session.

\section{DATA ANALYSIS}

To highlight the main effects of tACS over SI, data were collapsed into frequency bands according to previous electrophysiological studies (Pfurtscheller, 1981; Yordanova and Kolev, 1998; Benoit et al., 2000; Puligheddu et al., 2005; Kisley and Cornwell, 2006; Babiloni et al., 2008; Swettenham et al., 2009): delta (2-4 Hz), theta $(6-8 \mathrm{~Hz})$, alpha $(10-14 \mathrm{~Hz})$, beta $(16-20 \mathrm{~Hz})$, lower gamma $(22-30 \mathrm{~Hz})$, mid gamma $(32-50 \mathrm{~Hz})$, and high gamma $(52-70 \mathrm{~Hz})$. A one-way repeated measures analysis of variance (ANOVA) on the tactile sensation ratings (ranging from 0 to 3 ), with seven levels of frequency bands, was carried out both for the training and for the full experiment. Greenhouse-Geisser correction was applied when necessary to compensate for the violation of the sphericity assumption. In the presence of significant interactions, corrected pairwise comparisons were performed by Fisher's LSD test, in order to limit experiment-wise error rate to $\alpha$, and to maximize the power of the test detecting pairwise differences. We took $p<0.05$ as the criterion for statistical significance.

\section{RESULTS}

In the main experiment, repeated measures ANOVA revealed a significant main effect of frequency $F(4.34,47.76)=4.257$, $\mathrm{MSE}=0.695, p=0.004$. Post hoc comparisons showed that tACS produced a stronger tactile sensation when tACS was delivered at an alpha frequency $(10-14)$ than at delta $(p=0.015)$, theta $(p=0.003)$, mid gamma $(p=0.022)$. Furthermore, high gamma tACS also induced stronger tactile sensations than delta $(p=0.038)$ and theta tACS $(p=0.014)$. Although the effect is weaker, slightly stronger tactile sensation was reported at beta frequency as compared to theta frequency $(p=0.033$; Figure 1). 
The same pattern of results was observed in the training session (see Appendix), although repeated measures ANOVA on the training session did not show a significant main effect of stimulation frequency. This could be attributable to the fact that in the initial training session, the participants could not reliably distinguish the tACS-induced tactile sensation due to lack of experiences with such an unusual sensation. However, the pattern of results was consistent with the main experiment: tactile sensation induced by alpha or high gamma was rated as stronger than other stimulation frequencies (see Appendix).

\section{DISCUSSION}

In the present study, we found that tACS over the somatosensory cortex induced tactile sensations in a frequency-dependent manner. This phenomenon was reported in the left hand, contralateral to the site of stimulation in the right S1. This sensation was particularly strong when alpha or high gamma frequency stimulation was used. A minor effect of beta stimulation was also observed (Figure 1).

Our results indicate that tACS can induce sensations via its influences on cortical activity. tACS is a relatively unexplored method of brain stimulation and issues of volume conduction are underexplored. For example, in a previous study that stimulated over the visual cortex to induce visual sensations (phosphenes; Kanai et al., 2008), it remained possible that phosphenes could be induced by stimulation of the retina due to volume conduction of current over the scalp (Schwiedrzik, 2009; Schutter and Hortensius, 2010). The concern of peripheral stimulation is alleviated in our current study, because the tactile sensation was induced in the distant contralateral hand and this cannot be easily attributed to volume conduction of current. These results suggest that tACS is effective at directly interacting with the cortical activity in the somatosensory cortex through the frequency-dependent brain stimulation.

The induction of tactile sensation was most effective at alpha and high gamma frequencies. These two frequencies have been found to be correlated to the sensory-motor activity as reported by Pfurtscheller and Neuper's (1992) group, with an initial desynchronization of alpha oscillations indicating the planning of a movement, accompanied by a subsequent generation of gamma oscillations just before the onset of a movement.

Alpha stimulation was the most effective frequency band for inducing tactile sensations. Previous scalp EEG, MEG, and ECoG studies consistently showed that alpha band activity in the somatosensory cortex exhibits event-related desynchronization that is a brief decrease in power in response to the contralateral tactile stimulation (Leocani et al., 1997; Crone et al., 1998; Cheyne et al., 2003; Del Percio et al., 2010; Dockstader et al., 2010). It may appear paradoxical that tactile sensation is induced by alpha tACS, because alpha band activity in the somatosensory cortex (i.e., the $\mu$ rhythm) is typically associated with the resting state and it decreases in response to tactile stimulation (Pfurtscheller et al., 1996). One possible explanation for the induction of tactile sensation by alpha frequency stimulation is that tACS may interfere with ongoing resting state activity. During the experiment, participants were in the resting state without any direct tactile stimulation to their hand. If tACS were delivered at a frequency near the $\mu$ rhythm of the participants, it may have caused resonance-like interferences with the ongoing cortical activity. Such an effect could enhance the oscillation amplitude of the $\mu$ rhythm to an unusual level and could be perceived by participants as tactile sensation. Indeed,

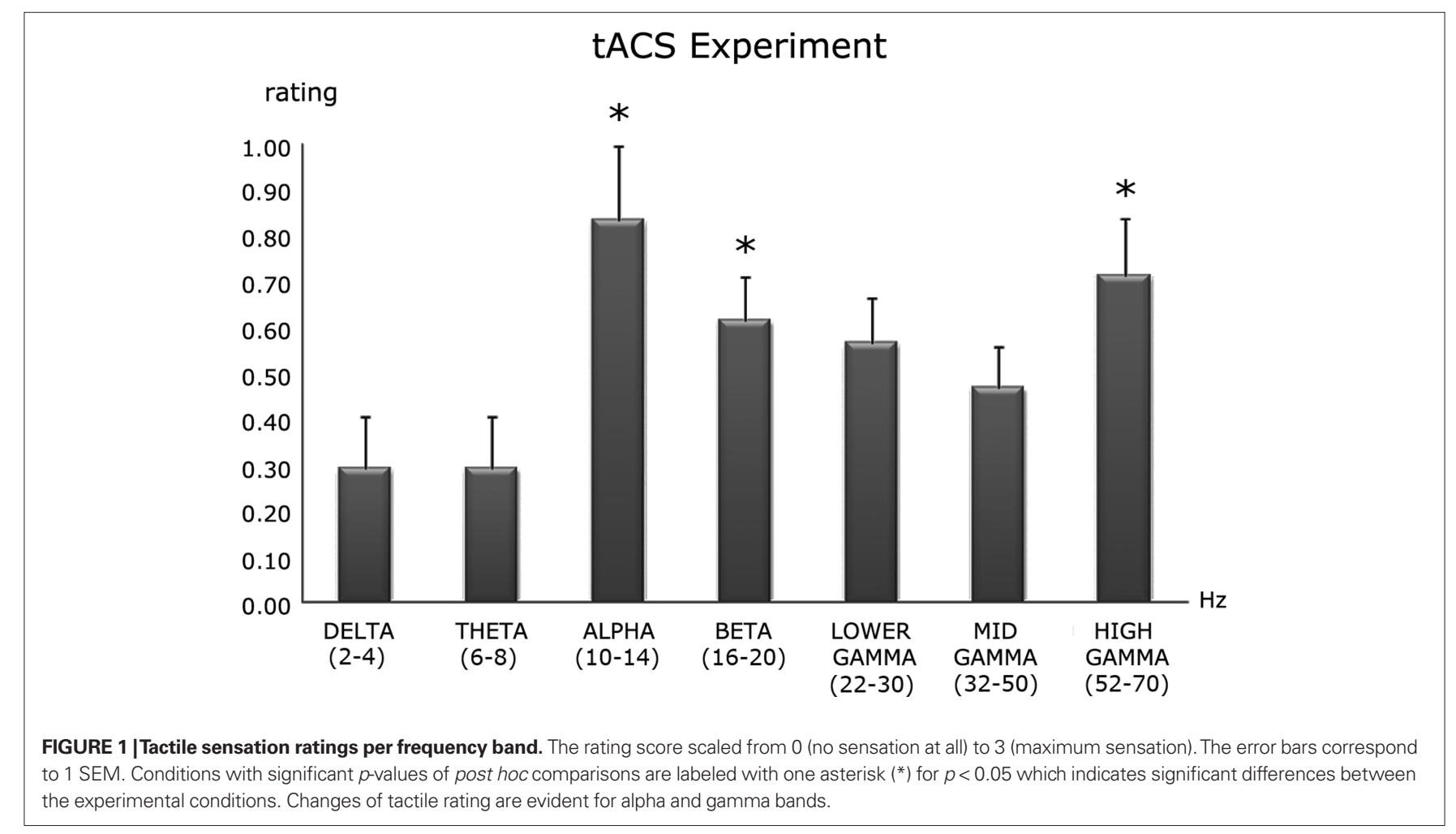


the power of ongoing alpha band activity prior to weak tactile stimulation has been shown to predict successful detection of the stimuli (Linkenkaer-Hansen et al., 2004; Jones et al., 2007). Thus, possible modulation of ongoing $\mu$ rhythm with alpha tACS might artificially enhance tactile sensitivity even in the absence of stimulation to the hand. A similar interpretation was put forward in a previous study that used alpha tACS to induce phosphenes (Kanai et al., 2008). In that study, alpha frequency was the most effective stimulation for phosphene induction, when tACS was delivered over the visual cortex in the dark. The occipital alpha is known to become more dominant in the absence of visual input, much like the enhanced $\mu$ rhythm in the somatosensory cortex in a resting state. Thus, it is conceivable that tACS is effective at inducing sensations when applied at a frequency near the most dominant frequency in a given state.

High gamma frequency $(50-70 \mathrm{~Hz})$ was another effective tACS frequency for inducing tactile sensation. High gamma responses are frequently observed in SI following proprioceptive (Arnfred et al., 2007) and tactile stimulation (Kanayama and Ohira, 2009; Kanayama et al., 2009) and this response is enhanced when attention is directed to the tactile stimulation (Bauer et al., 2006). This suggests that high gamma in SI is directly linked with tactile sensation. The tACS at a high gamma frequency in our study may have directly induced cortical activity similar to the high gamma response observed in previous MEG studies (Arnfred et al., 2007; Betti et al., 2009; Cebolla et al., 2009), thereby inducing the tactile sensation. While the previous studies observed correlations between evoked high gamma response and tactile sensation, our present study provides a direct causal link between the two, that is, delivery of high gamma frequency current to SI produces subjective tactile sensation.

On rare occasions, participants reported tactile sensations outside the target contralateral hand. For instance, they sometimes reported a tactile sensation of the neck and of the leg. This might indicate technical limitations related to the focality of tACS as have already been reported for similar stimulation techniques such as transcranial direct current stimulation (tDCS; Wagner et al., 2007). The exact pattern of electrical field induced inside the skull depends on the anatomical features such as the morphology of an individual's gyri and sulci (Datta et al., 2008). Thus, even though we localized tACS target SI with TMS, possible variability in the intracranial current flow could contribute to stimulation of neighboring somatosensory regions. MRI-guided targeting combined with a ring electrode as proposed in Datta et al. (2009) may improve the focality of tACS and reduce inter-individual variability of the stimulated site. Another important issue for the future is to establish how inter-individual differences in peak EEG frequencies relate to direct brain stimulation (Klimesch, 1999; Babiloni et al.,
2004; Thut and Miniussi, 2009). On the other hand, in this study, a large frequency spectrum was explored in order to establish the reliability of the tactile effects.

Our results showed that the strongest tactile sensation was reported when tACS was delivered at alpha frequency. The rating was on average around 0.85 , which is even weaker than the rating of " 1 " indicating participants felt a faint tactile sensation. This suggests the induced tactile sensation was on average rather subtle. However, our results indicate that the pattern of frequency dependency was highly consistent both across subjects (Figure 1) and across two sessions (Figure A1 in Appendix). Given the fact that subjects were blind to stimulation frequency, our results indicate that tACS had frequency-dependent effect on the induction of tactile sensation.

There are at least two possible reasons why the induced sensations were so weak. Firstly, the current intensity can be too weak to reliably activate the somatosensory cortex. The current density over the stimulation electrode was $62.5 \mu \mathrm{A} / \mathrm{cm}^{2}$, and this is rather high within our current (conservative) safety limit. However, it is plausible that higher current intensity is needed to deliver current to the cortex transcranially. While we expect clearer sensations will be evoked by tACS with higher intensity, this possibility cannot be tested easily due to missing safety criterions and possibly uncomfortable sensation on the skin under the electrode. Secondly, the optimal stimulation frequency may vary across individuals. Individual differences in the peak frequency of each band have been reported in various EEG studies. If optimal stimulation frequency is sharply tuned for individual's EEG frequency, averaging reports across off-peak frequencies would lower the mean ratings of tactile sensation. Thus, it will be an important future study to compare individual's EEG frequency and optimal stimulation frequency.

In summary, we have shown that tACS over human somatosensory cortex can induce tactile sensation in a frequencydependent fashion. tACS was most effective when delivered at alpha or high gamma frequency. These findings further consolidate the efficacy and frequency specificity of tACS (Marshall et al., 2006; Kanai et al., 2008; Kirov et al., 2009; Pogosyan et al., 2009) and link previously reported frequency specific neural responses in the somatosensory cortex with subjective experiences of tactile sensation.

\section{ACKNOWLEDGMENTS}

All the Department of Clinical Neurophysiology staff (Göttingen, Germany), Dr. Giulia Galli for assistance with data collection (ICN, UCL, London, England). Vincent Walsh is supported by Royal Society and the Medical Research Council. Ryota Kanai is supported by Human Frontier Science Program.

\section{REFERENCES}

Arnfred, S. M., Hansen, L. K., Parnas, J., and Morup, M. (2007). Proprioceptive evoked gamma oscillations. Brain Res. 1147, 167-174.

Babiloni, C., Babiloni, F., Carducci, F., Cappa, S., Cincotti, F., Del, P. C., Miniussi, C., Moretti, D. V., Pasqualetti,
P., Rossi, S., Sosta, K., and Rossini, P.M. (2004). Human cortical EEG rhythms during long-term episodic memory task. A high-resolution EEG study of the HERA model. Neuroimage 21, 1576-1584.

Babiloni, C., Vecchio, F., Bares, M., Brazdil, M., Nestrasil, I., Eusebi, F., Rossini, P.
M., and Rektor, I. (2008). Functional coupling between anterior prefrontal cortex (BA10) and hand muscle contraction during intentional and imitative motor acts. Neuroimage 39 1314-1323.

Bauer, M., Oostenveld, R., Peeters, M., and Fries, P. (2006). Tactile spatial attention enhances gamma-band activity in somatosensory cortex and reduces lowfrequency activity in parieto-occipital areas. J. Neurosci. 26, 490-501.

Benoit, O., Daurat, A., and Prado, J. (2000). Slow $(0.7-2 \mathrm{~Hz})$ and fast $(2-4$ $\mathrm{Hz}$ ) delta components are differently correlated to theta, alpha and beta 
frequency bands during NREM sleep. Clin. Neurophysiol. 111, 2103-2106.

Betti, V., Zappasodi, F., Rossini, P. M., Aglioti, S. M., and Tecchio, F. (2009). Synchronous with your feelings: sensorimotor \{gamma\} band and empathy for pain. J. Neurosci. 29, 12384-12392.

Cebolla, A. M., De, S. C., Bengoetxea, A., Leurs, F., Balestra, C., d'Alcantara, P., Palmero-Soler, E., Dan, B., and Cheron, G. (2009). Movement gating of beta/gamma oscillations involved in the N30 somatosensory evoked potential. Hum. Brain Mapp. 30, 1568-1579.

Cheyne, D., Gaetz, W., Garnero, L., Lachaux, J.P., Ducorps,A., Schwartz, D., and Varela, F. J. (2003). Neuromagnetic imaging of cortical oscillations accompanying tactile stimulation. Brain Res. Cogn. Brain Res. 17, 599-611.

Crone, N. E., Miglioretti, D. L., Gordon, B., Sieracki, J. M., Wilson, M. T., Uematsu, S., and Lesser, R. P. (1998). Functional mapping of human sensorimotor cortex with electrocorticographic spectral analysis. I. Alpha and beta event-related desynchronization. Brain 121(Pt 12), 2271-2299.

Datta, A., Elwassif, M., Battaglia, F., and Bikson, M. (2008). Transcranial current stimulation focality using disc and ring electrode configurations: FEM analysis. J. Neural Eng. 5, 163-174.

Datta, A., Elwassif, M., and Bikson, M. (2009). Bio-heat transfer model of transcranial DC stimulation: comparison of conventional pad versus ring electrode. Conf. Proc. IEEE Eng. Med. Biol. Soc. 2009, 670-673.

Del Percio, C., Infarinato, F., Iacoboni, M., Marzano, N., Soricelli, A., Aschieri, P., Eusebi, F., and Babiloni, C. (2010). Movement-related desynchronization of alpha rhythms is lower in athletes than non-athletes: a high-resolution EEG study. Clin. Neurophysiol. 121, 482-491.

Dockstader, C., Cheyne, D., and Tannock, R. (2010). Cortical dynamics of selective attention to somatosensory events. Neuroimage 49, 1777-1785.

Engel,A. K., Fries, P., and Singer,W. (2001). Dynamic predictions: oscillations and synchrony in top-down processing. Nat. Rev. Neurosci. 2, 704-716.

Fries, P. (2005). A mechanism for cognitive dynamics: neuronal communication through neuronal coherence. Trends Cogn. Sci. 9, 474-480.

Jones, S. R., Pritchett, D. L., Stufflebeam, S. M., Hamalainen, M., and Moore, C.
I. (2007). Neural correlates of tactile detection: a combined magnetoencephalography and biophysically based computational modeling study. J. Neurosci. 27, 10751-10764.

Kanai, R., Chaieb, L., Antal, A., Walsh, V. and Paulus, W. (2008). Frequencydependent electrical stimulation of the visual cortex. Curr. Biol. 18, 1839-1843.

Kanai, R., Paulus, W., and Walsh, V. (2010). Transcranial alternating current stimulation (tACS) modulates cortical excitability as assessed by TMSinduced phosphene thresholds. Clin. Neurophysiol. 121, 1551-1554.

Kanayama, N., and Ohira, H. (2009). Multisensory processing and neura oscillatory responses: separation of visuotactile congruency effect and corresponding electroencephalogram activities. Neuroreport 20, 289-293.

Kanayama, N., Sato, A., and Ohira, H. (2009). The role of gamma band oscillations and synchrony on rubber hand illusion and crossmodal integration. Brain Cogn. 69, 19-29.

Kirov, R., Weiss, C., Siebner, H. R., Born, J., and Marshall, L. (2009). Slow oscillation electrical brain stimulation during waking promotes EEG theta activity and memory encoding. Proc. Natl. Acad. Sci. U.S.A. 106, 15460-15465.

Kisley, M.A., and Cornwell, Z. M. (2006). Gamma and beta neural activity evoked during a sensory gating paradigm: effects of auditory, somatosensory and cross-modal stimulation. Clin. Neurophysiol. 11, 2549-2563.

Klimesch,W. (1999). EEG alpha and theta oscillations reflect cognitive and memory performance: a review and analysis. Brain Res. Rev. 29, 169-195.

Leocani, L., Toro, C., Manganotti, P., Zhuang, P., and Hallett, M. (1997). Event-related coherence and eventrelated desynchronization/synchronization in the $10 \mathrm{~Hz}$ and $20 \mathrm{~Hz}$ EEG during self-paced movements. Electroencephalogr. Clin. Neurophysiol. 104, 199-206.

Linkenkaer-Hansen, K., Nikulin, V. V., Palva, S., Ilmoniemi, R. J., and Palva, J. M. (2004). Prestimulus oscillations enhance psychophysical performance in humans. J. Neurosci. 24 10186-10190.

Llinas, R. R., and Steriade, M. (2006) Bursting of thalamic neurons and states of vigilance. J. Neurophysiol. 95, 3297-3308.
Marshall, L., Helgadottir, H., Molle, M. and Born, J. (2006). Boosting slow oscillations during sleep potentiates memory. Nature 444, 610-613.

Nitsche, M. A., Doemkes, S., Karakose, T., Antal, A., Liebetanz, D., Lang, N., Tergau, F., and Paulus, W. (2007). Shaping the effects of transcranial direct current stimulation of the human motor cortex. J. Neurophysiol. 97, 3109-3117.

Paulus, W. (2010). On the difficulties of separating retinal from cortical origins of phosphenes when using transcranial alternating current stimulation (tACS). Clin. Neurophysiol. 121 987-991.

Pfurtscheller, G. (1981). Central beta rhythm during sensorimotor activities in man. Electroencephalogr. Clin. Neurophysiol. 51, 253-264.

Pfurtscheller, G., and Neuper, C. (1992) Simultaneous EEG $10 \mathrm{~Hz}$ desynchronization and $40 \mathrm{~Hz}$ synchronization during finger movements. Neuroreport 3, 1057-1060.

Pfurtscheller, G., Stancak, A. Jr., and Neuper, C. (1996). Event-related synchronization (ERS) in the alpha band - an electrophysiological correlate of cortical idling: a review. Int J. Psychophysiol. 24, 39-46.

Pogosyan, A., Gaynor, L. D., Eusebio, A., and Brown, P. (2009). Boosting cortical activity at beta-band frequencies slows movement in humans. Curr. Biol. 19, 1637-1641.

Puligheddu, M., de Munck, J. C., Stam, C. J., Verbunt, J., de Jongh, A., van Dijk, B. W., and Marrosu, F. (2005). Age distribution of MEG spontaneous theta activity in healthy subjects. Brain Topogr. 17, 165-175.

Rohracher, H. (1935). Über subjektive Lichterscheinungen bei Reizung mit Wechselströmen. Z. Sinnesphysiol. 66, 164-181.

Schutter, D. J., and Hortensius, R. (2010) Retinal origin of phosphenes to transcranial alternating current stimulation. Clin. Neurophysiol. 121 , 1080-1084.

Schwarz, F. (1947). Über die elektrische Reizbarkeit des Auges bei Hell- und Dunkeladaptation. Pflugers Arch. 249, 67-86.

Schwiedrzik, C.M. (2009). Retina or visua cortex? The site of phosphene induction by transcranial alternating current stimulation. Front. Integr. Neurosci. 3:6 doi: 10.3389/neuro.07.006.2009

Swettenham, J. B., Muthukumaraswamy, S. D., and Singh, K. D. (2009). Spectral properties of induced and evoked gamma oscillations in human early visual cortex to moving and stationary stimuli. J. Neurophysiol. 102 , 1241-1253.

Thut, G., and Miniussi, C. (2009). New insights into rhythmic brain activity from TMS-EEG studies. Trends Cogn. Sci. 13, 182-189.

Wagner, T., Valero-Cabre, A., and PascualLeone, A. (2007). Noninvasive human brain stimulation. Annu. Rev. Biomed. Eng. 9, 527-565.

Yordanova, J., and Kolev, V. (1998). Eventrelated alpha oscillations are functionally associated with P300 during information processing. Neuroreport 9, 3159-3164.

Yousry, T. A., Schmid, U. D., Alkadhi, H., Schmidt, D., Peraud, A., Buettner, A., and Winkler, P. (1997). Localization of the motor hand area to a knob on the precentral gyrus. A new landmark. Brain 120(Pt 1), 141-157.

Zaehle, T., Rach, S., and Herrmann, C. S. (2010). Transcranial alternating current stimulation enhances individual alpha activity in human EEG. PLoS One 5, e13766. doi: 10.1371/journal. pone.0013766

Zaghi, S., de Freitas, R. L., de Oliveira, L. M., El-Nazer, R., Menning, S., Tadini, L., and Fregni, F. (2010). Inhibition of motor cortex excitability with 15 $\mathrm{Hz}$ transcranial alternating current stimulation (tACS). Neurosci. Lett. 479, 211-214.

Conflict of Interest Statement: The authors declare that the research was conducted in the absence of any commercial or financial relationships that could be construed as a potential conflict of interest.

Received: 10 November 2010; accepted: 13 January 2011; published online: 02 February 2011

Citation: Feurra M, Paulus W, Walsh V and Kanai $R$ (2011) Frequency specific modulation of human somatosensory cortex. Front. Psychology 2:13. doi: 10.3389/ fpsyg.2011.00013

This article was submitted to Frontiers in Perception Science, a specialty of Frontiers in Psychology.

Copyright () 2011 Feurra, Paulus, Walsh and Kanai. This is an open-access article subject to an exclusive license agreement between the authors and Frontiers Media $S A$, which permits unrestricted use, distribution, and reproduction in any medium, provided the original authors and source are credited. 


\section{APPENDIX}

\section{RESULTS OF TRAINING EXPERIMENT}

Although the data from the training session did not show statistically significant frequency dependency $[F(6,66)=0.574, \mathrm{MSE}=0.097$, $p=0.750]$, the similar pattern of frequency dependency as found in the main experiment is visible (Figure A1 in Appendix). This suggests that our participants could detect tACS induced tactile sensation more reliably after a short training session.

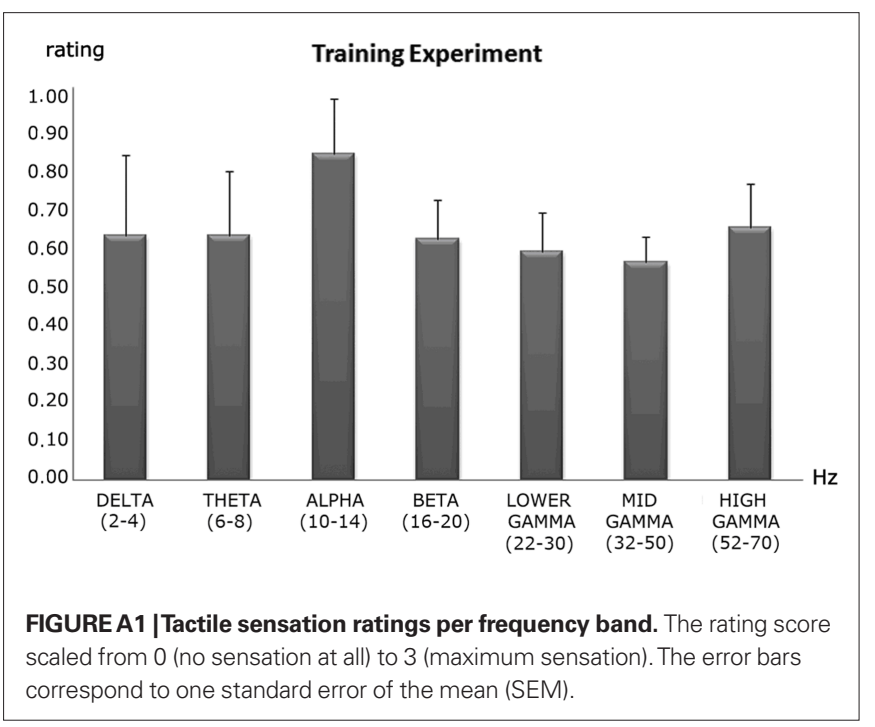

